\title{
Adaptações agudas promovidas por exercícios no aumento da expressão gênica, conteúdo e translocação da proteína GLUT-4 no músculo esquelético e melhora na responsividade à insulina Acute adaptations promoted by exercises upon GLUT-4 translocation, gene and protein content in the skeletal muscle and improvement in insulin sensitivity
}

Henrique Quintas Teixeira Ribeiro*, Rodolfo Gonzalez Camargo*, Waldecir Paula Lima**, Ricardo Zanuto***, Luiz Carlos Carnevali Junior****

${ }^{*}$ Grupo de Biologia Molecular da Célula, Instituto de Ciências Biomédicas I, Universidade de São Paulo/SP, **Grupo de Biologia Molecular da Célula, Instituto de Ciências Biomédicas I, Universidade de São Paulo/SP, Instituto Federal de Educação, Ciência e Tecnologia de São Paulo/SP, ${ }^{* * *}$ Laboratório de Sinalização Intracelular, Instituto de Ciências Biomédicas I, Universidade de São Paulo/SP, ${ }^{* * * * F a c u l d a d e ~ A n h a n g u e r a ~ d e ~ T a b o a ̃ o ~ d a ~ S e r r a / S P, ~ G r u p o ~ d e ~ B i o l o g i a ~ M o l e c u l a r ~ d a ~ C e ́ l u l a, ~ I n s t i t u t o ~ d e ~ C i e ̂ n c i a s ~ B i o m e ́-~}$ dicas I, Universidade de São Paulo/SP

\section{Resumo}

Diversos estudos indicam que os exercícios físicos em geral promovem melhorias fisiológicas tanto em indivíduos saudáveis quanto em portadores de patologias. Dentre estas melhorias, citase o aumento da expressão do GLUT-4, promovendo aumento da tolerância à glicose em indivíduos que apresentam resistência à insulina, como diabetes, particularmente de tipo 2. Nesta revisão, será apresentada uma descrição de vários estudos sobre o tema, destacando os efeitos agudos promovidos por treinamentos aeróbios e anaeróbios, particularmente relacionados ao aumento da sensibilidade à insulina. Foi realizada uma vasta revisão bibliográfica de artigos internacionais indexados ao Pubmed, entre os meses de janeiro e julho de 2010.

Palavras-chave: exercícios físicos, GLUT-4, diabetes, insulina.

\begin{abstract}
Several studies indicate that physical exercise in general promotes physiological improvements in both healthy subjects and in patients with pathologies. Among these improvements, the increased expression and amount of GLUT-4 may be described. Such adaptations clearly increase glucose tolerance in subjects with diabetes, particularly type 2 . In this review, a description of many studies on the subject will be showed, highlighting the acute effects provided by aerobic and anaerobic training, particularly related to the increase of insulin sensitivity. We performed an extensive literature review of international articles indexed in Pubmed, between the months of January and July 2010.
\end{abstract}

Key-words: physical exercise, GLUT-4, diabetes, insulin. 


\section{Introdução}

Nos mamíferos, o sistema nervoso central necessita de glicose como fonte essencial de energia. Desta forma, para sobreviverem, indivíduos normais devem manter o nível plasmático de glicose entre 4-7 mM [1], através de alterações de mecanismos centrais e periféricos do organismo [2].

A insulina é um dos hormônios responsáveis por esta manutenção, sendo secretada pelas células beta-pancreáticas em resposta a um aumento da glicose plasmática. Nos tecidos musculares, assim como nos tecidos adiposos (em menor escala), a insulina aumenta a captação de glicose, diminuindo a produção de glicose hepática. Este hormônio atua também promovendo o armazenamento de substratos nos tecidos adiposos, no fígado e nos músculos, por estimular lipogênese e síntese de glicogênio e proteínas, respectivamente; além de inibir a lipólise, glicogenólise e proteólise [1], através de duas vias distintas de sinalização (citadas no próximo tópico). Em relação às células musculares esqueléticas, principal alvo de nosso estudo, pode-se afirmar que são os mais importantes alvos da insulina na regulação da glicose sanguínea [3]. Elas são responsáveis por pelo menos $80 \%$ da captação de glicose do sangue [4]. Rodnick et al. [5] reportaram que o GLUT-4 está presente em estruturas tubulovesiculares agrupadas no retículo transgolgi, e que através da insulina ou do exercício físico, são translocados até a membrana sarcoplasmática, onde ocorre a captação de glicose.

A diminuição da atividade muscular, que acarreta em mudanças no nível de expressão gênica dos receptores de glicose nas células musculares (GLUT-4), é um dos fatores que altera a sensibilidade à insulina nestas células [6]. Richter et al. e Tabata et al. $[7,8]$, através de seus estudos, apontaram que uma redução aguda no nível de atividades diárias, como, por exemplo, em imobilizaçôes de membros inferiores, ou até mesmo em um descanso deitado em uma cama, rapidamente diminuíram a sensibilidade à insulina dos músculos, bem como seu conteúdo de GLUT-4. Houmard et al. [9] mostraram que, em caso de um aumento do padrão de atividade física, como, por exemplo, no treinamento, ocorre aumento do conteúdo proteico de GLUT-4 nas células musculares.

Desta forma, pode-se esperar um aumento da sensibilidade à insulina, o que acarreta uma maior tolerância à glicose. Ainda seguindo esta linha de raciocínio, Kawanaka et al. [10] concluíram que a mudança da responsividade à insulina durante o destreinamento estava diretamente relacionada ao conteúdo de GLUT-4 muscular, e consequentemente quanto maior o aumento do conteúdo de GLUT-4 induzido pelo treinamento, tal efeito persiste por um período mais prolongado.

O conteúdo total de GLUT-4 tem sido considerado um dos fatores determinantes da responsividade a insulina na musculatura esquelética [11], fato extremamente importante, particularmente para indivíduos que apresentam quadro de resistência à insulina, como diabetes, obesidade e hipertensão. Além do aumento da expressão gênica e conteúdo de GLUT-4, o aumento da translocação das vesículas de GLUT-4 rumo à superfície celular, providenciado pelo aumento da atividade das proteínas envolvidas nesta cascata de sinalização, também é fundamental para uma maior responsividade à insulina [12]. Partindo desta premissa, o objetivo principal deste trabalho é revisar os estudos que apontam as modificações que o exercício realizado em diferentes intensidades promove em relação à diminuição da resistência à insulina, providenciada não só pelo aumento do conteúdo de GLUT-4, mas também pelo aumento da translocação de suas vesículas.

\section{Vias intracelulares da captação de glicose depen- dente $e$ independente de insulina}

A regulação da captação de glicose dependente de insulina pelas células musculares ocorre através da ativação de uma série de proteínas intracelulares [13]. A ligação da insulina com a subunidade alfa de seu receptor (IR) causa um aumento da atividade da tirosina kinase da subunidade beta, levando à autofosforilação deste receptor e à fosforilação do substrato-1 do receptor de insulina (IRS-1). A partir deste ponto, ocorre a sinalização de duas vias distintas, cujos finais se dão através da translocação de GLUT-4 para a membrana sarcoplasmática e para os túbulos T, captando a glicose através da difusão facilitada. Na primeira via, o IRS-1 se liga à subunidade regulatória do fosfatidilinositol 3-kinase (PI3-kinase), que ativa a subunidade catalítica 110 desta enzima.

A PI3-kinase catalisa a produçáo das metades dos fosfoinositídeos, que ativam as kinases dependentes dos fosfoinositídeos (PDK), incluindo a PDK-1. Um alvo desta cascata de sinalização é a Akt/proteína kinase B (Akt/PKB). A ativação destas proteínas resulta na translocaçáo do GLUT-4 [14]. Já a segunda via está relacionada ao gene $\mathrm{Cbl}$, que se encontra associado à proteína CAP [15]. Quando o Cbl é fosforilado, ocorre translocação do complexo Cbl-CAP, que recruta a proteína CrkII. Esta proteína também forma um complexo com a proteína $\mathrm{C} 3 \mathrm{G}$, que quando translocada, fosforila a proteína G da proteína TC10. A TC10, uma vez ativada, providencia um segundo sinal para o GLUT-4 (ver figura 1) [16].

O transporte de glicose pelas células musculares também é estimulado por mecanismos independentes de insulina devido a contraçôes musculares [17], por aumentar a ativação da AMP kinase (proteína 5' AMP-ativada kinase), uma enzima ativada pela diminuição da carga energética celular [18], que aumenta a translocação de GLUT-4 para a superfície celular. Um dos mecanismos responsáveis pela maior ativação da AMPK é o aumento nas razóes AMP/ATP e creatina/fosfocreatina [19]. A atividade contrátil altera o status energético das células musculares esqueléticas, e dependendo da intensidade das contraçôes pode haver diminuiçôes significantes nas concentraçóes de fosfocreatina e ATP, levando a uma maior ativação da AMPK [20]. Um outro mecanismo a ser citado é o aumento da concentração miocelular de cálcio devido à contração muscular [21]. 
A contração muscular é iniciada pela despolarização da membrana sarcoplasmática e dos túbulos $\mathrm{T}$, disparando o gatilho para a liberação de cálcio do retículo sarcoplasmático. O aumento intracelular de cálcio leva a uma interação entre os filamentos de actina e miosina, permitindo o desenvolvimento de tensão nas fibras. A CaMKK (cálcio/calmodulinadependente proteína kinase), uma molécula sensível ao cálcio ionizado, é ativada, e consequentemente aumenta a ativação da AMPK [22].

\section{Efeitos do exercício agudo no conteúdo de GLUT-4}

Embora o principal estímulo da secreçáo de insulina e glucagon seja a variação da glicemia, ambos hormônios também sofrem influência de sua secreção pelo sistema nervoso autônomo [23]. Mudanças na secreção pancreática de insulina e glucagon representam um importante papel na regulação da glicose plasmática [24]. É sabido que o pâncreas é inervado por nervos vagos e viscerais, e a estimulação destes nervos altera a secreçâo de insulina e glucagon [25].

A estimulação alfa adrenérgica inibe a secreção de insulina, enquanto que a estimulação beta adrenérgica estimula a liberação deste hormônio [26]. Durante o exercício, a estimulação dos nervos viscerais libera norepinefrina e inibe a secreção de insulina pelas células beta-pancreáticas [27]. A estimulação dos receptores alfa2 pela norepinefrina apresenta efeito inibitório na liberação de insulina, enquanto que a estimulação dos receptores beta adrenérgicos aumenta a liberação de insulina. No entanto, a ação da norepinefrina nos receptores alfa 2 predomina, e a secreçáo de insulina é reduzida [25].

A estimulaçáo elétrica do músculo ou uma série única de exercícios por corrida em esteiras ou natação mostraram um aumento da captação de glicose em diversos estudos, como revisado por Ivy [28]. O efeito agudo do exercício consiste em duas fases [29]. O efeito inicial dura por algumas horas e não necessita da presença de insulina. A duração desta fase parece estar correlacionada com a ressíntese de glicogênio e pode ser prolongada pela manipulação dietética, com o objetivo de adiar tal ressíntese [29]. Ainda em relação a esta fase, durante a atividade física ocorre um aumento da via de translocação de GLUT-4 devido ao aumento intracelular de cálcio. No momento em que ocorre a despolarização, fundamental para que haja interação entre os filamentos de actina e miosina, ocorre liberação do cálcio do retículo endoplasmático liso, que também atua como mediador do transporte de glicose. Holloszy et al. [30] observaram que a frequência da contração é responsável pelo aumento do transporte de glicose, não a duração, e nem tampouco a tensão do movimento.

No entanto, o estudo de Ihlemann et al. [31] apontou que em músculos sóleos incubados de ratos, a captação de glicose estimulada pela contração é mais dependente da produção de força do que da estimulaçáo da frequência. Este aumento de cálcio citoplasmático pode atuar iniciando ou facilitando a fosforilação de proteínas ou moléculas envolvidas nas cascatas de sinalização intracelulares, que desencadeiam os efeitos tanto agudos quanto crônicos do exercício em relaçáo ao transporte de glicose. Pode-se citar como exemplo desta fosforilação a proteína quinase $\mathrm{C}$ (cálcio dependente e sinalizadora intermediária), que devido à contração muscular é ativada, e parece estar envolvida na regulação do transporte de glicose que é estimulado por esta contração [32]. Na segunda fase, ocorre aumento da sensibilidade à insulina, que pode durar por até 24 horas ou até mesmo ao redor de 48 horas, dependendo da atividade realizada.

Annuzzi et al. [33] observaram o efeito de uma única sessão de exercício (3 horas, 50\% VO2 máx) em que após 24 horas a sensibilidade à insulina mantinha-se alta nos músculos que haviam sido utilizados. Etgen et al. [34], em um estudo em que ratos correram em uma esteira durante 5 dias/semana, entre 12-16 semanas, em que progressivamente atingiram velocidade de $32 \mathrm{~m} / \mathrm{min}$ após a oitava semana, mantendo esta velocidade posteriormente (estímulo intenso), apontaram que os efeitos deste treinamento em relação à responsividade à insulina ou à captação de glicose em músculos das patas traseiras (predominantemente compostos por fibras do tipo IIb) de ratos saudáveis apresentaram vida curta $(29 \%$ maior do que sedentários durante as primeiras 24 horas), e desapareceram dentro de 48 horas após o treinamento. Ren et al. [35] reportaram um grande aumento na expressáo gênica (duas vezes maior se comparado ao conteúdo de RNA-m de ratos controle) e no conteúdo de GLUT-4 no músculo epitrochlearis (músculo das patas frontais, compostos predominantemente por fibras glicolíticas) de ratos saudáveis (aproximadamente 1,5 vezes maior) 16 horas após uma sessáo de treinamento de natação ( 2 séries de 3 horas, sem carga acoplada, com 45 minutos de intervalo entre as séries), e apesar de não ter havido aumento na expressão gênica, houve aumento de aproximadamente duas vezes na quantidade de GLUT-4 16 horas após o fim de 2 sessôes, realizadas em dias consecutivos.

Kawanaka et al. [36], na tentativa de descobrirem se os efeitos do treinamento de natação em relação à responsividade à insulina poderiam ser mantidos por mais de 24 horas, submeteram um grupo de ratos a nadar 2 horas diárias, divididas em 4 séries de 30 minutos e separadas por um intervalo de 5 minutos, durante 5 dias. Após a primeira série, os ratos tinham anexado a seus corpos um peso correspondente a $2 \%$ de seus pesos corporais. O estudo apresentou como resultado que, após 18 horas após o treinamento, a responsividade à insulina e o conteúdo de GLUT-4 nos músculos epitrochlearis dos ratos aumentaram $85 \%$, e mantiveram-se $50 \%$ maior do que o observado nos ratos controle após 42 horas de treino.

Esses efeitos de treinamento retornaram ao nível controle após 90 horas após o treinamento. Após uma série aguda de exercício, o transporte de glicose nos músculos aumenta em um mesmo nível de um estímulo máximo de insulina. Durante o exercício agudo, é sabido que a ativação de AMPK estimula uma maior captação de glicose através da 
translocação do GLUT-4 até a superfície celular esquelética [37]. Neufer e Dohm [38] mostraram que a indução pelo exercício no aumento de GLUT-4 é mediada em nível transcricional. O RNA-m dos GLUT-4 e GLUT-1, bem como as próprias proteínas em si aumentaram nos músculos treinados e imagina-se que são responsáveis pelo aumento máximo da captação de glicose estimulado pela insulina. Zheng et al. [39] demonstraram que a expressão gênica do GLUT-4 é modulada em nível transcricional pela ativação da AMPK, fornecendo evidências adicionais do seu envolvimento na regulação da expressão gênica muscular devida ao exercício.

\section{Comparaçóes entre exercícios de diferentes inten- sidades na modulaçáo aguda da expressáo gênica e conteúdo de GLUT-4}

Terada et al. [40], em 2001, realizaram um estudo com a intenção de verificar os efeitos do treinamento de natação de altíssima intensidade no conteúdo do GLUT-4 dos músculos epitrochlearis de ratos saudáveis, bem como de comparar este conteúdo com o observado nos treinamentos de duração prolongada e intensidade baixa. $\mathrm{O}$ estudo demonstrou que o treinamento de altíssima intensidade, em que os ratos deveriam nadar 14 séries de 20 segundos nadando com 10 segundos de intervalo de descanso entre as séries, em um tempo total de 280 segundos, durante 8 dias, em que foi anexada aos corpos dos ratos uma carga equivalente a $14 \%$ de seus pesos corporais, levou a um aumento agudo de GLUT-4 semelhante ao aumento do treinamento de baixa intensidade, em que o grupo deveria nadar 2 séries de 3 horas de duraçáo, com intervalo de descanso de 45 minutos entre as séries, também durante 8 dias, em um tempo total de 360 minutos), respectivamente $83 \%$ e $91 \%$, que até entáo era tido como o estímulo mais forte para aumentar os níveis de GLUT-4 nos músculos epitrochlearis dos ratos [39].

Neste estudo, foram estudados 2 fatores que podem ter induzido um aumento da expressão gênica de GLUT-4: os fatores neurotróficos e a AMPK. Especulou-se que durante o treinamento de baixa intensidade houve maior liberação de fatores neurotróficos nos músculos epitrochlearis, embora essa taxa de secreção seja bem menor se comparada à taxa secretada durante o treinamento alta intensidade. É concebível a ideia de que em ambos os tipos de exercícios, a taxa secretada destes fatores foi suficientemente alta para induzir a expressão gênica de GLUT-4 nas células musculares de ratos. Em relação à AMPK, podem existir duas hipóteses distintas para a indução máxima da expressão gênica de GLUT-4. A primeira é baseada na afirmação de Hutber et al. [41], que apontou que a atividade da AMPK aumenta gradativamente durante o exercício. Desta forma, seria possível que durante o treinamento LIT haja um aumento considerável da atividade de AMPK, que acarreta em uma indução máxima da expressão gênica do GLUT-4.

A segunda hipótese é baseada em Rasmussen et al. [42], que demonstrou que a atividade da AMPK é dependente da intensidade do exercício. Sendo assim, é possível que no treinamento HIT ocorra uma ativação máxima da AMPK dentro de poucos minutos. Embora estas hipóteses sejam divergentes, em ambos os casos houve indução máxima da expressão gênica de GLUT-4.

\section{Conclusão}

O aumento da expressão gênica e do conteúdo do GLUT4, assim como a ativação das proteínas intracelulares envolvidas nas vias sinalizadoras de translocação das vesículas de GLUT-4, acarreta em um aumento à responsividade à insulina, algo favorável para indivíduos com quadro de resistência à insulina. Agudamente a prática de atividade física promove tanto o aumento da expressáo gênica quanto do conteúdo proteico, além de aumentar a translocação dos receptores de glicose nas células musculares, sendo que a natação, se comparada à corrida em esteira, é capaz de promover manutenção da diminuição à resistência à insulina por mais tempo. Atividades de alta intensidade e curta duração aparentemente são mais eficientes do que atividades de baixa intensidade e longa duração, por apresentarem menor tempo dispendido.

\section{Referências}

1. Saltiel AR, Kahn, CR. Insulin signaling and the regulation of glucose and lipid metabolism. Nature 2001;414(6865):799806.

2. Dallman MF, Akana SF, Bhatnagar S, Bell ME, Choi S, Chu A et al. Starvation: early signals, sensors, and sequelae. Endocrinology 1999;140:4015-23.

3. DeFronzo RA, Jacot E, Jequier E, Maeder E, Wahren J, Felber JP. The effect of insulin on the disposal of intravenous glucose. Results from indirect calorimetry and hepatic and femoral venous catheterization. Diabetes 1981;30:1000-7.

4. Kubo K, Foley JE. Rate-limiting steps for insulin-mediated glucose uptake into perfused rat hindlimb. Am J Physiol 1986;250(13):100-2.

5. Rodnick KJ, Slot JW, Studelska DR, Hanpeter DE, Robinson LJ, Geuze HJ, James DE. Immunocytochemical and biochemical studies of GLUT 4 in rat skeletal muscle. J Biol Chem 1992;267:6278-85.

6. Richter EA, Derave W, Wojtaszewski JF. Glucose, exercise and insulin: emerging concepts. J Physiol 2001;535:313-22.

7. Richter EA, Kiens B, Mizuno M, Strange S. Insulin action in human thighs after one-legged immobilization. J Appl Physiol 1989;67:19-23.

8. Tabata I, Suzuki Y, Fukunaga T, Yokozeki T, Akima H, Funato K. Resistance training affects GLUT-4 content in skeletal muscle of humans after 19 days of head-down bed rest. J Appl Physiol 1999;86:909-14.

9. Houmard JA, Hickey MS, Tyndall GL, Gavigan KE, Dohm GL. Seven days of exercise increase GLUT-4 protein content in human skeletal muscle. J Appl Physiol 1995;79:1936-38.

10. Kawanaka K, Tabata I, Katsuta S, Higuchi M. Changes in insulin-stimulated glucose transport and GLUT-4 protein in rat skeletal muscle after training. J Appl Physiol 1997;83:2043-47. 
11. Hansen PA, Gulve EA, Marshall BA, Gao J, Pessin JE, Holloszy JO, Mueckler M. Skeletal muscle glucose transport and metabolism are enhanced in transgenic mice overexpressing the GLUT-4 glucose transporter. J Biol Chem 1995;270:1679-84.

12. Jessen N, Goodyear LJ. Contraction signaling to glucose transport in skeletal muscle. J Appl Physiol 2005;99:330-7.

13. Shepherd PR, Kahn BB. Glucose transporters and insulin action. Implications for insulin resistance and diabetes mellitus. N Engl J Med 1999;341:248-57.

14. Henriksen EJ. Invited review: effects of acute exercise and exercise training on insulin resistance. J Appl Physiol 2002;93(2):788-96.

15. Ribon V, Saltiel AR. Insulin stimulates tyrosine phosphorylation of the proto-oncogene product of c-Cbl in 3T3-L1 adipocytes. Biochem J 1997;324:839-45.

16. Chiang SH, Baumann CA, Kanzaki M, Thurmond DC, Watson RT, Neudauer CL, et al. Insulin-stimulated GLUT4 translocation requires the CAP-dependent activation of TC10. Nature 2001;410:944-8.

17. Nesher R, Karl IE, Kipnis DM. Dissociation of effects of insulin and contraction on glucose transport in rat epitrochlearis muscle. Am J Physiol Cell Physiol 1985;249:226-32.

18. Kurth-Kraczek EJ, Hirshman MF, Goodyear LJ, and Winder WW. 5 AMP-activated protein kinase activation causes GLUT4 translocation in skeletal muscle. Diabetes 1999;48:1667-71.

19. Kemp BE, Mitchelhill KI, Stapleton D, Michell BJ, Chen ZP,Witters LA. Dealing with energy demand: the AMPactivated protein kinase. Trends Biochem Sci 1999;24:22-5.

20. Jessen N, Goodyear LJ. Contraction signaling to glucose transport in skeletal muscle. J Appl Physiol 2005;99(1)330-37.

21. Holloszy JO, Hansen PA. Regulation of glucose transport into skeletal muscle. Rev Physiol Biochem Pharmacol 1996;128:99193.

22. Jorgensen SB, Rose AJ. How is AMPK activity regulated in skeletal muscles during exercise? Front Biosc 2008;13:5589-604.

23. Woods SC, Chavez M, Park CR, Riedy C, Kaiyala K, Richardson $\mathrm{RD}$ et al. The evaluation of insulin as a metabolic signal influencing behavior via the brain. Neurosci Biobehav Rev 1996;20:139-44.

24. Wasserman DH. Regulation of glucose fluxes during exercise in the postabsorptive state. Annu Rev Physiol 1995;57:191-218.

25. Woods SC, Porte JD. Neural control of the endocrine pancreas. Physiol Rev 1974;54:596-619.

26. Luyckx AS, Lefebvre PJ. Mechanisms involved in the exerciseinduced increase in glucagons secretion in rats. Diabetes 1974;23:81-93.

27. Miller RE. Pancreatic neuroendocrinology: peripheral neural mechanisms in the regulation of the islets of Langerhans. Endocr Rev 1981;2:471-94.
28. Ivy JL. The insulin-like effect of muscle contraction. Exercise Sport Sci Rev 1987;15:29-51.

29. Garetto LP, Richter EA, Goodman MN, Ruderman NB. Enhanced muscle glucose metabolism after exercise in the rat: the two phases. Am J Physiol 1984;246:471-75.

30. Holloszy JO, Constable SH, Young DA. Activation of glucose transport in muscle by exercise. Diabetes Metab Rev 1986;1:409-24.

31. Ihlemann J, Ploug T, Hellsten Y, Galbo H. Effect of stimulation frequency on contraction-induced glucose transport in rat skeletal muscle. Am J Physiol Endocrinol Metab 2000;279:862-67.

32. Goodyear LJ, Kahn BB. Exercise, glucose transport and insulin sensitivity. Annu Rev Med 1998;49:235-61.

33. Annuzzi G, Riccardi G, Capaldo B, Kaijser L. Increased insulin-stimulated glucose uptake by exercised human muscles one day after prolonged physical exercise. Eur J Clin Invest 1991;21:6-12.

34. Etgen GJJr, Brozinick JTJr, Kang HY, Ivy JL. Effects of exercise training on skeletal muscle glucose uptake and transport. Am J Physiol 1993;264:723-33.

35. Ren JM, Semenkovich CF, Gulve EA, Gao J, Holloszy JO. Exercise induces rapid increases in GLUT-4 expression, glucose transport capacity, and insulin-stimulated glycogen storage in muscle. J Biol Chem 1994;269:14396-401.

36. Kawanaka K, Tabata I, Katsuta SE, Higuchi M. Changes in insulin-stimulated glucose and GLUT-4 protein in rat skeletal muscle after training. J Appl Physiol 1997;83(6):2043-47.

37. Holmes BF, Kurth-Kraczek EJ, Winder WW. Chronic activation of 5'-AMP-activated protein kinase increases GLUT-4, hexokinase, and glycogen in muscle. J Appl Physiol 1999;87(5)1990-5.

38. Neufer PD, Dohm GL. Exercise induces a transient increase in transcription of the GLUT-4 gene in skeletal muscle. Am J Physiol Cell Physiol 1993;265:1597-1603.

39. Zheng D, MacLean PS, Pohnert SC, Knigh JB, Olson AL, Winder WW, Dohm GL. Regulation of muscle GLUT-4 transcription by AMPK-activated protein kinase. J Appl Physiol 2001;90:1073-10.

40. Terada S, Yokozeki T, Kawanaka K, Ogawa K, Higuchi M, Ezaki $\mathrm{O}$, Tabata I. Effects of high-intensity swimming training on GLUT-4 and glucose transport activity in rat skeletal muscle. J Appl Physiol 2001;90(6):2019-24.

41. Hutber CA, Hardie DG, Winder WW. Electrical stimulation inactivates muscle acetyl-CoA carboxylase and increases AMPactivated protein kinase. Am J Physiol Endocrinol Metab 1997;272:262-66.

42. Rasmussen BB, Hancock CR, Winder WW. Post exercise recovery of skeletal muscle malonyl-CoA, acetyl-CoA carboxylase, and AMP-activated protein kinase. J Appl Physiol 1998;85:1629-34. 